\title{
Eau et mémoire chez Marie-Célie Agnant
}

\author{
Cremades-Cano, Isaac David
}

Universidad de Murcia, dicc1@um.es

\begin{abstract}
Resumen
La isla es un espacio constantemente evocado por la escritora haitiana Marie-Célie Agnant. A lo largo de su trabajo, los diferentes medios acuáticos tales como el océano y su inmensidad, el Mar Caribe que rodea la isla o las muchas lagunas, pero también los ríos y riachuelos, son elementos que parecen coexistir con en la misma amplitud en su tierra natal así como en su imaginación. Esta omnipresencia del líquido elemental constituye la esencia de un lenguaje poético original, en el que el agua adquiere una notable simbología. De hecho, El libro de Emma está repleto de descripciones, en las que los matices del color del agua del mar, azul en sus profundidades, a veces gris, naranja y roja al atardecer, ocultan fragmentos de la memoria colectiva del pueblo de Haití. Por otra parte, el río nos introduce al viaje interior de la protagonista, la memoria de sus antepasados africanos brota y se convierte en agua de río que fluye constantemente en sus novelas y poesías. Proponemos entonces profundizar en el análisis de la riqueza de estas metáforas que unen el agua a la memoria - individual y colectiva -, describiendo el viaje metafórico evocado a través del personaje de Emma, en su dimensión identitaria y femenina.
\end{abstract}

Palabras clave : Literatura de Haití ; Agnant ; Agua ; Memoria ; Identidad.

\section{Résumé}

L'île est un espace constamment évoqué par l'écrivaine haïtienne Marie-Célie Agnant. Dans l'ensemble de son œuvre, les différents milieux aquatiques tels que l'océan et son immensité, la mer des Caraïbes entourant l'île ou les nombreux lagons, mais aussi les fleuves et les petites rivières, sont des éléments qui semblent cohabiter avec la même ampleur dans son pays natal ainsi que dans son imaginaire. Cette omniprésence du liquide élémentaire constitue l'essence d'un langage poétique original, à partir duquel l'eau acquiert une remarquable symbolique. En effet, Le livre d'Emma est parsemé de descriptions où les nuances de la couleur de l'eau de la mer, bleue dans ses profondeurs, des fois grise, orange et rouge au coucher de soleil, cachent des bribes de la mémoire collective du peuple haïtien. D'ailleurs, le fleuve nous introduit au voyage intérieur d'Emma, la mémoire de ses ancêtres africaines jaillit et devient l'eau de rivière qui coule sans cesse dans son œuvre romanesque et poétique. Nous proposons donc d'approfondir l'analyse de la richesse de ces métaphores qui lient l'eau à la mémoire - individuelle et collective -, en décrivant le parcours métaphorique évoquée à travers le personnage d'Emma, dans sa dimension identitaire et féminine.

Mots-clés : Littérature haïtienne ; Agnant ; Eau ; Mémoire ; Identité.

\begin{abstract}
The island is a space constantly evoked by the Haitian writer Marie-Celie Agnant. Throughout her work, different aquatic environments such as the ocean and its immensity, the Caribbean Sea surrounding the island or the many lagoons, but also rivers and streams are elements that seem to coexist with the same amplitude in her homeland and in her imagination. This omnipresence of elemental liquid is the essence of an original poetic language, in which the water acquires a striking symbolism. Indeed, The Book of Emma is full of descriptions where the nuances of the colour of the water of the sea, blue in its deepness, sometimes grey, orange and red at sunset, hiding bits of the collective memory of the Haitian people. Furthermore, the river brings us to Emma's inner journey, the memory of her African ancestors springs and becomes river water flowing constantly in her novels and poems. Then, we propose to deepen the analysis of the
\end{abstract}


wealth of these metaphors that bind water to the memory - individual and collective -, describing the metaphorical journey evoked through the character of Emma in her identity and gender dimensions.

Keywords : Haitian literature ; Agnant ; Water ; Memory ; Identity.

\section{Introduction}

L'eau des rivières, des lagons, de la pluie mais surtout de la mer s'intègre dans les récits antillais sous formes diverses. Si l'on considère qu' ' un récit est un récit antillais à cause de l'inclusion de la nature. » (Bruno, 2005: 25), le développement d'une symbolique associée à l'eau enrichie spécialement le langage poétique de ces écrivains. L'omniprésence de l'élément aquatique dans le paysage insulaire est donc, source d'inspiration qui sert à exprimer, audelà de la description réaliste, des traits historiques et culturels. Nabil A. Boudraa, dans son étude consacrée à la poétique du paysage, propose justement une interprétation qui nous aide à déterminer le point de vue adoptée dans ce travail :

Le paysage est en fait le lieu où l'espace géographique parcouru par l'individu (et par extension la communauté) [...]. La constitution de son imaginaire, de sa culture et de sa parole en dépend. Ce rapport au lieu n'est un rapport d'appartenance ou de propriété mais de mémoire et d'existence (2002 : 4).

Dans la quête identitaire entreprise par ces auteurs postcoloniaux, ces paysages aquatiques deviennent un outil particulier pour réveiller ces fragments d’une mémoire blessée, ne faisant pas encore partie de l'Histoire de ces peuples dominés pendant des siècles par les Européens. De cette manière, c'est au moyen de l'eau que l'écrivaine d'origine haïtienne Marie-Célie Agnant délivre la mémoire du personnage principale de Le livre d'Emma. En fait, l'eau semble devenir le fils conducteur et le véhicule de communication de cette mémoire douloureuse remémoré par Emma tout au long du récit, en assumant donc cette lutte contre l'oubli et, spécialement, contre le long silence de la voix féminine, comme exprime également l'écrivaine d'origine guadeloupéenne G. Pineau :

Ici-là, en ce temps où la mémoire s’éveille et se retourne sur le passé, ces femmes sortent de l’ombre et marchent dans les traces ouvertes de l'histoire. [...] elles n'ont plus peur et disent à leurs arrière-petites-filles qu'il est temps de rompre les silences, temps de renverser les mémoires et de revêtir les habits de l’Histoire (1998: 13).

En effet, M-.C. Agnant met en scène, de la même façon que les écrivaines caribéennes francophones Maryse Condé, Simone Schwarz-Bart, Myriam Warner-Vieyra ou Gisèle Pineau notamment, une sorte de mémoire hybride composée, d'un côté, de cette mémoire proprement des femmes, «À un moment où les femmes affirmaient leur position dans le monde. Il va sans dire que les Antillaises avaient elles aussi, le besoin de faire entendre leur voix et de faire connaître leurs revendications. » (Leticee, 2003 : 6), et d'un autre côté d'un type de mémoire que Carmen Mata définit à l'aide de Maurice Bloch ${ }^{1}$ comme : "[...] "la mémoire historico-sémantique" [...] qui se nourrit des transmissions de souvenirs et des recherches des historiens, investit le champ de l'esclavage et du marronnage [...]. » (2013: 106). Ces deux mémoires semblent s'entrecroiser et confluent pour la construction d'un passé-présent plus authentique, puisqu'il s'agit d'une inestimable contribution qui complète et complémente les réalités décrites à travers la narrative, avec une mise en valeur de la voix féminine : «Résultat de l'accession de maintes Antillaises à l'indépendance économique ou intellectuelle, la voix féminine devient profuse [...]. » (Antoine, 1992 : 362), comme affirmait déjà aux années 90 ce spécialiste en littératures francophones des Antilles.

${ }^{1}$ De l'étude de M. Bloch (1995). « Mémoire autobiographique et mémoire historique du passé éloigné », in Enquête 2, p. 59-79. 


\section{Les souvenirs d'une vie face à une mémoire générationnelle au féminin}

Accusée du meurtre de son bébé, Emma est internée dans un hôpital psychiatrique à l'attente d'une évaluation, qui détermine si elle est apte à subir le procès judiciaire. Étant donnée sa négative à s'exprimer dans la langue des anciens colonisateurs, son médecin doit demander les services d’un interprète de créole : Flore. Elle va donc rencontrer une Emma, froide et réticente d'abord, qui va progressivement la captiver. En fait, Flore semble être emportée par un fort courant d'eau lorsqu'Emma fouille dans sa mémoire :

Je ne suis plus une simple interprète. Petit à petit, j’abandonne mon rôle, je deviens une partie d’Emma, j’épouse le destin d'Emma. Au cours de cette quatrième séance, j'ai l'impression que mon esprit quitte la chambre et s'en va voguer sur le fleuve en compagnie d’Emma. C’est alors, je crois, que j’ai pris la décision de la suivre jusqu'au bout. (Agnant, $2001: 18)$

En effet, Flore commence à connaître les habitudes d'Emma qui passe son temps face à la fenêtre de sa chambre « les yeux rivés sur le fleuve » (Agnant, 2001 : 17). Plongée dans ses pensées, Emma semble dissoudre les quatre murs de sa chambre grâce à une sorte de déplacement psychologique qui lui procure observer le mouvement de l'eau. Cet élément du paysage ne devient pas seulement le fils conducteur dans le procès de remémoration d’Emma, mais aussi un mécanisme d’évasion efficace, comme décrit Flore : «Emma contemple le fleuve, comme si elle voulait mettre en place un puzzle. Des morceaux de glace flottent à la surface de l'eau. Elle paraît avoir oublié le temps, elle nous a oubliés » (Agnant, 2001 : 19).

Même si l'eau qui coule symbolise traditionnellement le passage inéluctable du temps, Emma semble ne pas s'en apercevoir, immergée dans cet état d’esprit, et devient une femme mystérieuse qui garde un grand secret. L’eau en mouvement suit le lit du fleuve, en traçant un chemin sur lequel le liquide circule comme s'il s'agissait d'un voyageur qui parcourt le paysage. Alors les termes eau et voyage se rapprochent et montrent une dimension physique ainsi que psychologique. Le mouvement dans l'espace évoqué par le fleuve sert enfin à représenter symboliquement le voyage à l'intérieur de la conscience d'Emma :

Contre la fenêtre, elle appuie son front étroit et promène ses yeux farouches sur les arbres dans le parc, les ramène vers la droite sur le fleuve qui, au loin, roule ses eaux brunâtres. Puis elle vient à nous, s’avance vers un fauteuil dans lequel elle s’affaisse, le corps las, comme si elle venait de parcourir une si longue distance. (Agnant, 2001 : 11).

La fenêtre de la chambre close le paysage comme le cadre d'un tableau qui, en cette occasion semble prendre vie dans l'esprit d’Emma. La double dimension de ce voyage est suscitée avec le mouvement du regard mystérieux, ne laissant pas entrevoir les pensées d’Emma, et la comparaison finale qui fait référence à une fatigue plutôt physique.

Cette conception symbolique du voyage est aussi partagée par un autre personnage qui fait partie des souvenirs de la vie d’Emma. En effet, Baptiste, le mari Rosa, sa grand-mère maternelle, arrive également à s’évader de sa malheureuse réalité grâce à l’eau, à cette occasion, de la mer : « Souvent, elle devait aller le quérir au bord de la mer, sur le rivage. Elle le trouvait assis, le regard cloué sur les vagues, un regard qui flottait » (Agnant, 2001 : 113). La matérialisation de ce regard en mouvement perpétuel et l'acquisition de la flottabilité lui confèrent la faculté d'entreprendre ce voyage imaginaire, qu'Emma pratique souvent depuis la fenêtre de sa chambre. Alors, la mer aussi bien que le fleuve sont l'outil idéal pour s'évader et évoquer un passé plus ou moins lointain. Ce passé semble partager avec l’eau, sa propriété de dissoudre, dans ce cas, une réalité qui finalement les rattrape.

L'eau devient donc un moyen d'évasion qui permet aux personnages de remémorer et, à Emma spécialement, de reproduire non seulement les souvenirs d'une vie mais aussi le passé ancestral qui s'entrecroise, des voix qui reculent jusqu’aux générations africaines :

Grand-Lagon, ce bout de terre accroupi au milieu de l’océan, Grand-Lagon, faut pas avoir peur de le dire, c'est une terre de malédiction, Emma. Cette eau qui la baigne depuis le jour de sa naissance, cette eau, dans son bleu si bleu, cache des siècles de sang vomi des cales des négriers, sang de tous ces nègres que l'on jetait par-dessus bord. C'est 
ainsi que la malédiction est entrée. Elle s'est infiltrée dans l'eau des rivières, dans celle que nous buvons, elle s'est mêlée à notre sang, l’a corrompu. (Agnant, 2001 : 112)

L'eau se mêle au sang dans ce passé métis reproduit dans ce récit. La mémoire générationnelle d’Emma se traduit donc par une sorte de chronique de l'histoire collective du peuple haïtien d'origine africaine, mais aussi l'histoire au féminin, qui implique sortir de la marginalité ces voix longtemps silencieuses, une multivoix de toute une lignée de femmes, d'après l'analyse proposé par Lucie Lequin : « dans une seule voix narrative, M-.C. Agnant en fait entendre plusieurs et passe de la mémoire individuelle à la mémoire collective dans un mouvement de va-et-vient entre les deux. » (2002: 23).

C'est à travers la formation qu'Emma essaie d'échapper du paradigme raciste et machiste qui pousse ces voix féminines à l'oubli. Ainsi, dans sa thèse du doctorat, elle avoue des faits jamais racontés dans les chroniques officielles : «Blancs, Nègres, moins Blancs, moins Nègres, tous se jetaient sur les femmes couleur de nuit, sans leur demander leur avis, comme s'ils puisaient l'eau de la rivière pour étancher leur soif. » (Agnant, 2001 : 135). Le recours à l'image de l'eau comme élément généreux et synonyme de gratuité offerte par la nature, rend cette comparaison sa juste cruauté.

\section{Eau et bleu, vie et mort}

L'eau marque tant la naissance d'Emma dans tout ce bleu, que l'origine collective de tout un peuple, surgi après l'enlèvement et la traversée de l'océan. Elle fait référence aux tonalités bleues de l'eau de son île natale : « le bleu intense qui enserre en permanence un lambeau de terre abandonnée au milieu de l'océan. » (Agnant, 2001 : 7). Avec cette rude description qui se limite à un morceau de terre, une terre déchirée, détachée et un adjectif associé à cet espace insulaire, M.-C. Agnant décrit un pays qui traverse de grandes difficultés, en mettant en relief que son isolation géographique n’est pas la seule cause de cette condition. En conséquence, la situation socio-économique haïtienne, dégradée en permanence avec la grave crise chronique qui s'est installée dans le pays, est étroitement associée à un caractère très particulier du paysage : l’immensité de la couleur bleue qui renvoie le ciel sur l'eau de la mer. Ainsi explique Emma au docteur :

La première chose que l'on voit quand on vient au monde à Grand-Lagon, c'est le bleu. Certains disent, là-bas, que l'intensité du bleu cause une manière de folie... [...] C’est à cause du bleu, docteur. Il a toujours été autour de GrandLagon, comme la désespérance. Au fond, il est là pour brouiller la pérennité de la désespérance. À Grand-Lagon, on pourrait dire bleu, comme on dit désespérance (2001 : 20).

Par contre, les métaphores liées à la mer et à sa couleur ont le pouvoir de remémorer le fait antagonique de la naissance : « la profondeur du bleu a une gravité, solennelle, supra-terrestre. Cette gravité appelle l'idée de la mort. » (Chevalier \& Gheerbrant, 1982 : 129). Avec cette riche symbolique de l'eau, M-.C. Agnant réussit à ressusciter une multitude d'esclaves, ses ancêtres africains, qui n'étaient pas enterrés mais submergées dans ce procès d'humiliation continuelle infligé par les Européens. Cette nuance historico-culturelle comprise dans ce sens figuré attribue à la mer un caractère parfaitement identitaire. En fait, la mer pendant le voyage dans les cales négrières et puis les fonds océaniques entourant l’île, servent à évoquer une nécropole aquatique au risque d'être oubliée.

Ce rapprochement de la mer à la mort, au voyage et le recours à cet emplacement hypothétique du cimetière de l'opprimé, sont également repérables dans le travail d'autres auteurs et même au moyens d'autres disciplines artistiques différentes de la littérature, telles que la peinture, la sculpture et la photographie. Ainsi affirme également Bachelard dans son essai L'eau et les rêves: "L'imagination profonde, l'imagination matérielle veut que l'eau ait sa part dans la mort ; elle a besoin de l'eau pour garder à la mort sons sens de voyage. » $1991: 104)$.

Comme c'est le cas de l'œuvre du photographe sous-marin et sculpteur d'origine guyanaise Jason de Caires Taylor, par exemple, The Silent Evolution (2010) : à travers un ensemble de 400 sculptures humaines à taille réelle submergées, il semble matérialiser une idée similaire à celle d'Emma avec une mise en valeur des fonds marins, non seulement pour leur conservation mais surtout pour leur rôle à l'époque de l'esclavage. Les visages de ces sculptures, entourés de tout ce bleu, rouvrent cette dimension historique des fonds marins où, même s'ils ont étés endommagés par la contamination et frappés 
par des phénomènes météorologiques, la vie réapparaît malgré ce passé dévastateur. En fait, le corail et tout un écosystème renaît sur ces sculptures telles que la société haïtienne qui survie et continue à lutter, sous diverses formes, malgré les grandes difficultés.

L'œuvre acquiert, en somme, un grand pouvoir évocateur qui transmet un message plutôt optimiste : « Taylor’s art is like no other, a paradox of creation, constructed to be assimilated by the ocean and transformed from inert objects into living breathing coral reefs, portraying human intervention as both positive and life-encouraging ${ }^{2}$. »

Pour le peintre martiniquais Bruno Pédurand, ce liquide reprend également cette dimension historique douloureuse : « Différents éléments (silhouettes, barques) renvoient aussi à la tragique traversée des esclaves. Dans des tons ocre, terre, rouges, ses peintures symboliques ouvrant sur plusieurs niveaux d’interprétation. » (Berthet, 2009 : 313). Dans ce monde onirique de ses peintures, l'eau n'est plus claire et bleue, mais elle devient obscure et se mélange à la terre pour représenter ce passage à l'autre vie, telle que l'eau qui est sous la barque de Caron. Alors, l'eau et la mort réapparaissent ensemble dans une métaphore où l'eau s'imprègne d'un caractère symbolique, qui est à la fois un trait identitaire.

En littérature, par exemple, un des personnages de la célèbre auteure guadeloupéenne M. Condé réfléchit devant la mer : « Assis sur le pont supérieur, Razyé regardait les travées de la mer et se disait quel beau suaire cela ferait, cette étoffe de deuil moutonnant à perte de vue. » (1995 : 246). Les termes « suaire » et « étoffe » et l'association du bleu avec le deuil renforcent l'image de la mort, de même que son caractère éternel est lié à la ligne de l'horizon qui dessine l'océan.

La mer et ses profondeurs peuvent donc abriter une mémoire du passé et de l'esclavage, mais aussi les souvenirs du présent : le drame actuel de la migration que souffrent beaucoup d'haïtiens forcés à entreprendre. Alors, le paysage maritime et ses eaux sont encore subjectivisés avec le but d’exprimer les parallélismes entre les conflits intérieurs des personnages et l'environnement liquide qui les conduit vers un futur incertain, comme décrit ce personnage du roman En attendant la montée des eaux de M. Condé :

La mer c'est terrible !

Quel que soit l'endroit où tu te places pour la considérer, elle est pareille. Ce n'est pas comme un paysage qui est soit beau soit laid, avec des parties distinctes. [...] C'est partout une couleur identique, des vagues qui moutonnent pareillement, par-ci, par-là, avec des plaques d'un blanc sale de l’écume. (2010:60).

Ce personnage haïtien exprime ses sentiments pendant la traversée clandestine, où la monotonie de la mer calme devient antithèse de la peur et du désespoir qu'il ressent et partage avec les autres passagers. Dans cet extrait, l'océan et son bleu omniprésent est en quelque sorte un personnage aussi, car il joue un rôle fondamental dans l'avenir de ces personnages et de leur île. Cependant, cette calme apparente peut rapidement se tourner en menace et montrer une facette tout à fait opposée, comme décrit un autre personnage de M. Condé dans le point intermédiaire d'une traversée d'une île à une autre, juste au moment où ils ne divisent aucune côte : « Des paquets d'eau détrempent le pont. À perte de vue, la mer était creusée de gueules de monstres qui semblaient n’avoir qu'une envie, engloutir le navire. » (1995 : 322-323). Cette métaphore accorde encore une fois un aspect meurtrier à la mer, mais il est intéressant d'ajouter comment l'océan symbolise une transition, dans ce cas liée à des traits identitaires des Antillais : l’exile et l’émigration.

À travers l'eau, M-.C. Agnant exprime également la fin du parcours sur terre, une vie qui s’achève, celle d’Emma : « Son âme a rejoint le fleuve pour entreprendre le voyage de retour. » (Agnant, 2001 : 164). Ainsi, elle fait encore référence à la «mémoire historico-sémantique » qui soude Emma avec son passé ancestral africain éternellement: «Elle disait toujours, elle disait sans cesse qu'elle reprendrait un jour la route des grands bateaux. » (Agnant, 2001 : 163). Démunie de sa réalité diasporique, basée sur l'incompréhension et la souffrance, la mort ne semble la libérer qu'après avoir transmis tout son héritage mémoriel : « la descente vers l’océan est le rassemblement des eaux, le retour à l’indifférenciation, l’accès au Nirvâna » (Chevalier \& Gheerbrant, 1982 : 355), instrument de la libération définitive d’Emma.

\footnotetext{
${ }^{2}$ Biographie sur le site web de l'auteur $<\underline{\text { http://www.underwatersculpture.com/about/biography/ }>}$
} 


\section{Conclusions}

Nous pouvons constater le développement d'un imaginaire autour de l'eau, élément qui devient un outil logiquement essentiel pour exprimer certains traits identitaires des Haïtiens et, par extension, de tous les Antillais et AfricainsAméricains. L'analyse de l'intériorisation de cet élément dans le récit d'Emma ne fait que remettre en question le passé et le présent des haïtiens en exile.

Si la culture de tradition orale et la mémoire familiale se transmettait de génération en génération, Emma doit changer cet ordre ancestrale et léguer ses savoirs à une femme n’appartenant pas à la lignée de sa famille. Ces deux femmes, Emma et Flore, en apparence très éloignées l'une de l'autre, incarnent donc la lutte contre le silence et l'oubli respectivement. Avec un processus similaire, cette littérature haïtienne grandit dans le monde actuel sous la forme de littérature migrante d'expression française au Canada. Alors, l'association traditionnelle entre littérature et son lieu de création n'est plus opératoire dans ce cas précis.

Il semble donc s'établir un parallélisme entre ces deux changements de l'ordre classique, car ils supposent une vraie rupture qui permet, nonobstant, la survie d'une identité haïtienne complète liée à l'eau. D'un côté, il y a une transmission désarticulée de la mémoire et, d'un autre, l'existence d'une littérature disloquée en essence. Ces deux éléments, vertèbres du travail de l'Agnant militante et engagée, aident à redéfinir sa propre identité - et par extension, celle du peuple haïtien et sa diaspora -. Il nous semble, en somme, la solution face à l'oubli du passé, ainsi que la lutte contre les difficultés et les complexités inhérentes à l’Haïti du présent.

\section{Références bibliographiques}

Abraham, Marie et Pineau, Gisèle (1998). Femmes des Antilles. Traces et voix. Paris : Stock.

Antoine, Régis (1992). La littérature franco-antillaise. Paris : Karthala.

Agnant, Marie-Célie (2001). Le livre d’Emma. Montréal : Mémoire.

BACHELARD, Gaston (1991). L'eau et les rêves. Paris : José Corti.

Berthet, Dominique (2009). « Bruno Pédurand » dans Conseil régional de la Guadeloupe. Anthologie de la Peinture en Guadeloupe, des origines à nos jours. Paris : HC Éditions. Pp. 312-320

BOUDRAA, Nabil Augustin (2002). La poétique du paysage dans l'œuvre d'Édouard Glissant, Kateb Yacine et William Faulkner.

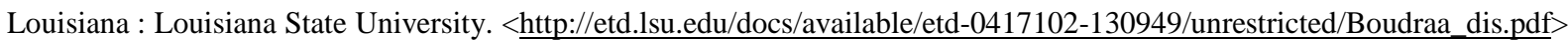
[Consulté en janvier 2016]

BRuno, Kimberly (2005). Corps et nature chez trois écrivaines guadeloupéennes. Ontario: McMaster University.

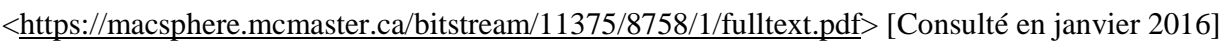

Chevalier, Jean et GHEERBrant, Alain (1982). Dictionnaire des symboles. Mythes, rêves, coutumes, gestes, formes, figures, couleurs, nombres. Paris : Robert Laffont.

CondE, Maryse (1995). La migration de cœurs. Paris : Robert Laffont.

CONDE, Maryse (2010). En attendant la montée des eaux. Paris : Lattès.

LETICEE, (2003). «Résistance antillaise au féminin?» dans Labrys, études féministes, $\mathrm{n}^{\circ} 3$. <http://www.labrys.net.br/labrys3/web/fran/leticee2.htm> [Consulté en février 2016]

Lequin, Lucie (2002). « Marie-Célie Agnant : une écriture de la mémoire et du silence. » dans Marc Maufort et Franca Bellarsi. Reconfigurations : Canadian literatures and postcolonial identities. Bruxelles : Peter Lang. Vol. VII, p. 22-32.

MATA, Carmen (2013). «La mémoire de l’esclavage et de la représentation dans l'œuvre de Marie-Célie Agnant. Mémoire identitaire, mémoire vivante » dans Colette Boucher et Thomas C. Spear. Paroles et silences chez Marie-Célie Agnant. L'oublieuse mémoire d'Haïti. Paris : Karthala. P. 105-119.

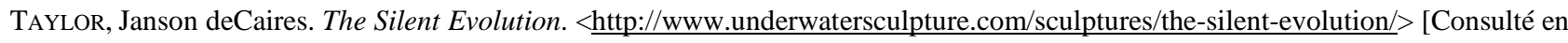
mars 2016] 